\title{
Detectors for particle identification
}

\author{
Peter Križan ${ }^{a, b}$

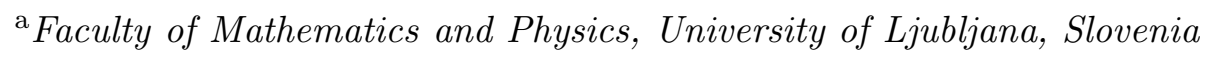 \\ b Jožef Stefan Institute, Ljubljana, Slovenia
}

\begin{abstract}
The paper reviews recent progress in particle identification methods. A survey of motivations and requirements for particle identification in various experimental environments is followed by the main emphasis, which is on the recent development of Čerenkov counters, from upgrades of existing devices to a novel focusing radiator concept and new photon detectors. The impact of including a precise measurement of the time of arrival of Cerenkov photons to increase the kinematical region over which particle identification can be performed is discussed. The progress in dedicated time-of-flight counters with recently developed very fast single photon detectors is also evaluated.
\end{abstract}

Key words: Ring imaging Čerenkov counter, proximity focusing, Aerogel, Belle, BaBar, HERA-B, focusing radiator

PACS: 29.40.Ka

\section{Introduction}

One of the main driving forces of the research and development of particle identification methods in the last decade was the need to have excellent hadron identification for precision $B$ physics measurements. For a statistically significant measurement of $\mathrm{CP}$ violation in the $B$ system, the tagging of $B$ meson flavour with the kaon charge was an indispensable method. In addition, for the study of rare few body hadronic decays of $B$ mesons it is essential to separate pions from kaons up to the kinematic limits of the experiment. Reliable hadron identification is also important in the search for quark-gluon plasma and in studies of the nucleon structure.

Email address: peter.krizan@ijs.si (Peter Križan). 
Hadrons are identified by their mass, which is, in turn, determined by combining the measurements of momentum and velocity. Assuming that momentum is inferred from the measured radius of curvature in magnetic field, the remaining issue is to measure the velocity with a sufficient precision. This is either achieved by measuring the time-of-flight, ionization losses or Čerenkov angle of the particle. In the present contribution recent progress in Cerenkov counters and time-of-flight measurements is discussed.

The structure of the paper is as follows. We first review the Cerenkov counters in some of the running or recently completed experiments. A number of new methods is presented, from upgrades of existing devices to a novel focusing radiator concept and new photon detectors. We then discuss the benefits of including a precise measurement of the time of arrival of Cerenkov photons in order to reduce the dispersion error and the even more ambitious use in a combined time-of-flight (TOF) and time-of-propagation (TOP) counter. Finally, we will discuss the progress in dedicated TOF counters with recently developed very fast photon detectors.

\section{2 Čerenkov counters}

\subsection{DIRC at BaBar}

The DIRC (Detector of Internally Reflected Cherenkov light) of the BaBar spectrometer is a special type of a ring-imaging Čerenkov counter (1), based on the detection of Cerenkov photons trapped in the quartz radiator bar (Fig. 1). The patterns on the photon detector are quite complicated, but result in well resolved peaks in the Cerenkov angle distribution. The time of arrival of photons is used to eliminate background from conversion events in the water tank, to assign photons to proper tracks, and to eliminate most of the ambiguities in the photon-track reconstruction.

The basic performance parameters of the counter are in excellent agreement with expectations (1). The single photon resolution amounts to $9.6 \mathrm{mrad}$. The number of photons depends on the polar angle of the charged track, but always stays above 20 for $\beta=1$ particles. The efficiency for kaon identification exceeds $90 \%$ in the momentum range $0.5 \mathrm{GeV} / c-3 \mathrm{GeV} / c$, while the probability that a pion is identified as a kaon stays at a few percent level (Fig. 2). 

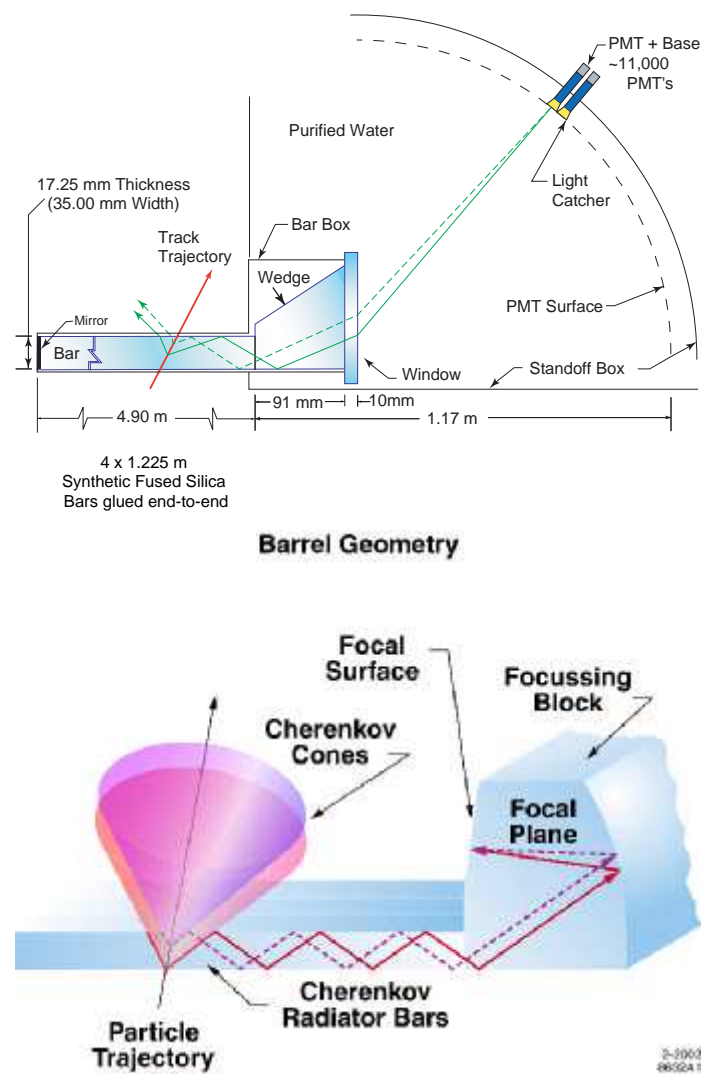

Fig. 1. Principle of the DIRC counter. (top) and its upgrade, the focusing DIRC (bottom).

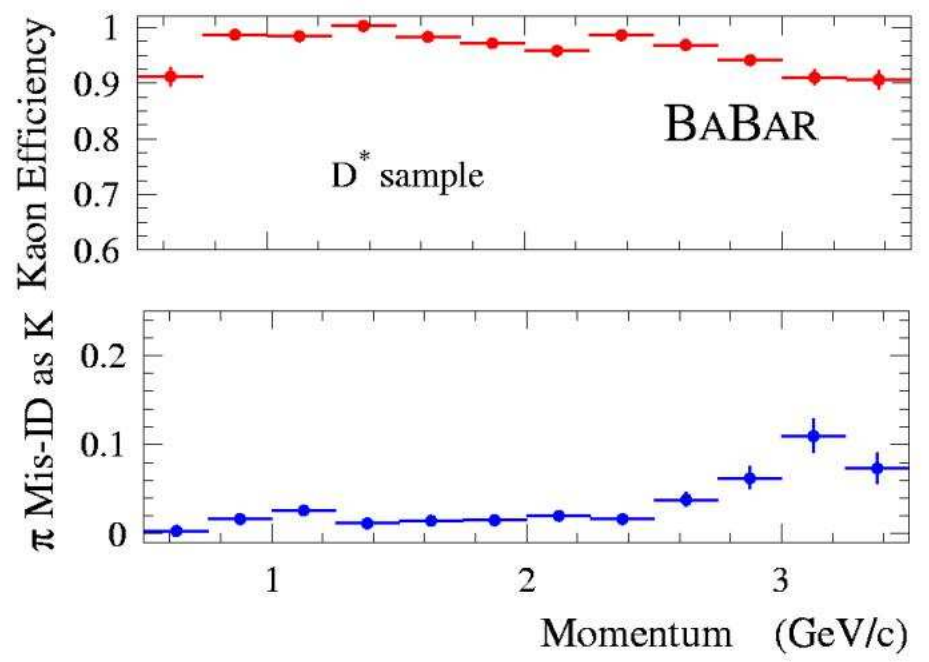

Fig. 2. Particle identification with DIRC: $K$ efficiency and $\pi \rightarrow K$ misidentification probability. 


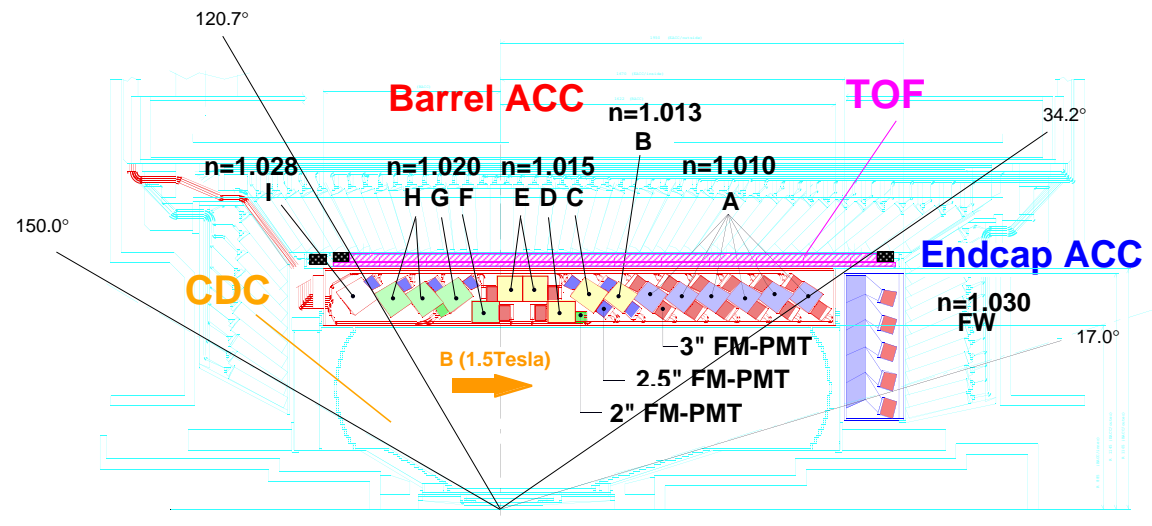

Fig. 3. Aerogel Čerenkov Counter (ACC) of the Belle spectrometer.

\subsection{ACC at Belle}

In the Belle spectrometer the separation of kaons from pions is performed with the Aerogel Cherenkov Counter (ACC), a threshold Čerenkov detector (Fig. 3) with aerogel as radiator (2). The refractive index of the radiator is chosen so that pions emit Čerenkov light, while kaons stay below threshold. Since the momentum spectrum of particles becomes harder in the forward direction, the refractive index of the modules gradually decreases from $n=1.028$ to $n=1.01$. Note that while in the central (barrel) part it is possible to measure both the tagging kaons and the $B \rightarrow \pi \pi, K \pi$ decay products, only the former can be identified in the forward (end-cap) direction. The kaon identification efficiency amounts to $90 \%$ with the pion fake probability equal to $6 \%$ (3).

\subsection{HERA-B RICH}

The HERA-B collaboration was the first to employ multianode photomultiplier tubes in a RICH counter (Fig. 4) (4), after having shown that the Hamamatsu R5900 PMTs (versions M16 and M4 with 16 and 4 square shaped channels, respectively) have a capability to detect single photons with high efficiency, and little cross-talk (5). The photon detector performed very well, showing clear rings with very few noisy channels $(<0.5 \%)$ even in a very hostile environment of a hadron machine, as can be seen from Fig. 5.

A common drawback of vacuum based photon detectors is a rather large fraction of dead area. While for single channel PMTs reflective cones can be used, multichannel PMTs need an imaging system. In the HERA-B RICH a system 


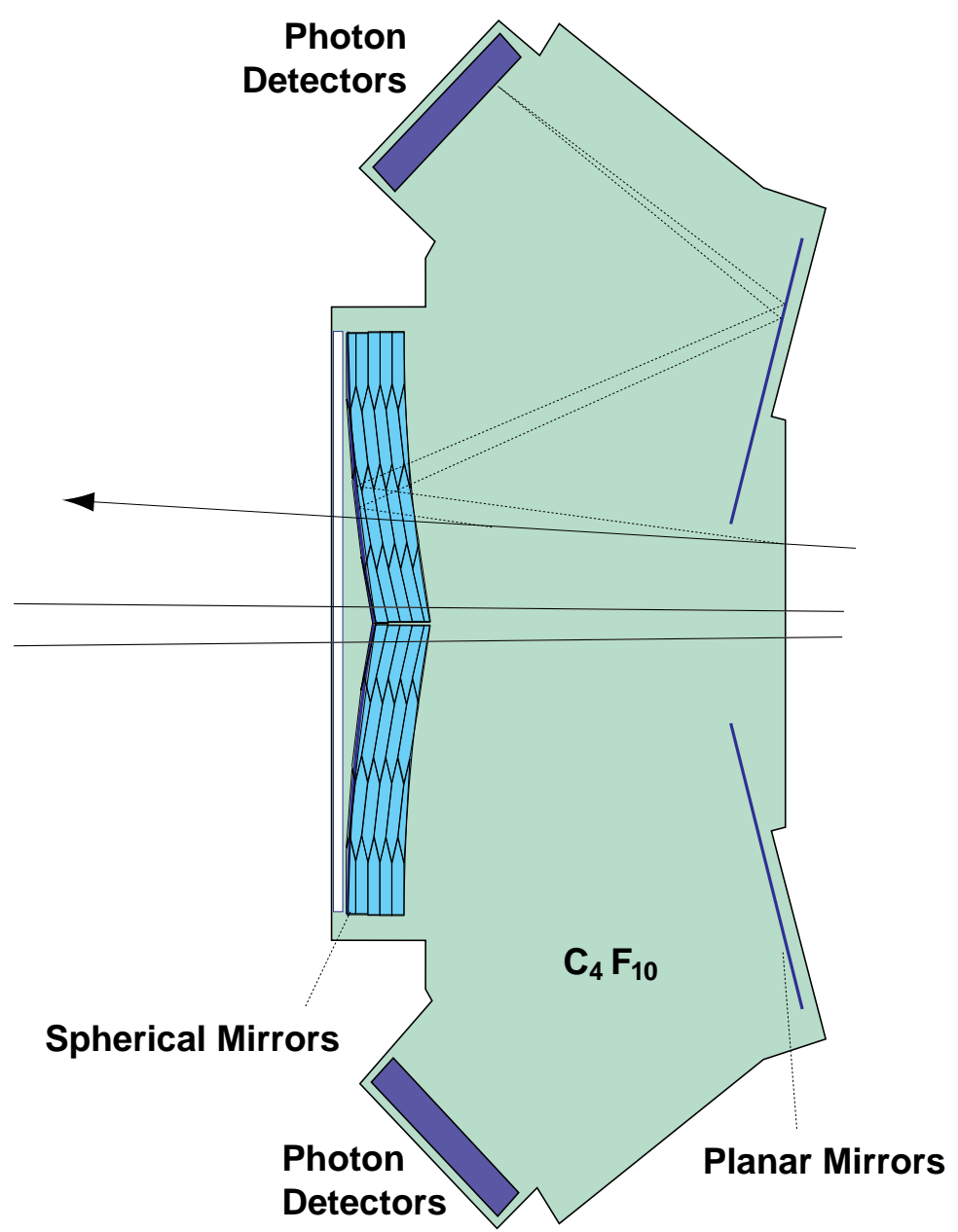

Fig. 4. Layout of the HERA-B RICH counter.

of two lenses, shown in Fig. 6, was used to demagnify the image on the focal surface by a factor of 2 (6). The system of lenses is used both to reduce the dead area, as well as to adapt the required granularity of the photon detector to the granularity of the multianode PMT. The transparency of the system is high in the region of high quantum efficiency of the tube. It also has a flat acceptance for photons with incidence angles below $140 \mathrm{mrad}$ as required by the detector geometry.

The experience of the HERA-B collaboration shows that a RICH counter can safely be operated even at high track densities with counting rates exceeding $1 \mathrm{MHz}$ per channel in the hottest part of the photon detector (4). A typical event is shown in the lower plot of Fig. 5. No degradation of performance has been observed over the five years of operation. The relevant RICH parameters were determined from the data. On average 32 photons were detected per $\beta=1$ ring. The single photon resolution was $0.7 \mathrm{mrad}$ in the finer granularity area covered by R5900-M16 PMTs. All parameters are in very good agreement with expectations. The particle identification capabilities are illustrated in Fig. 7. 

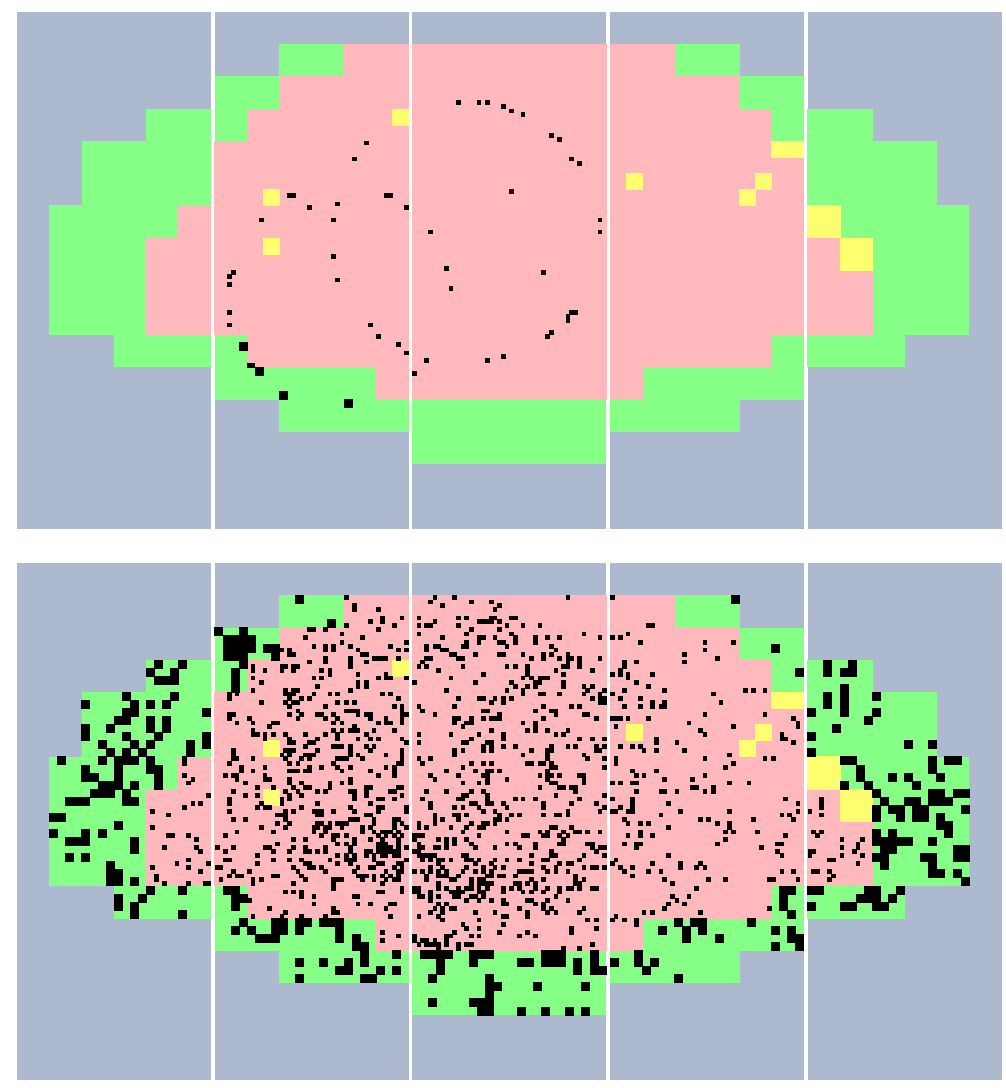

Fig. 5. Events recorded in the lower half of the HERA-B RICH: a low multiplicity event (top) with two clear rings, and a typical event (bottom).

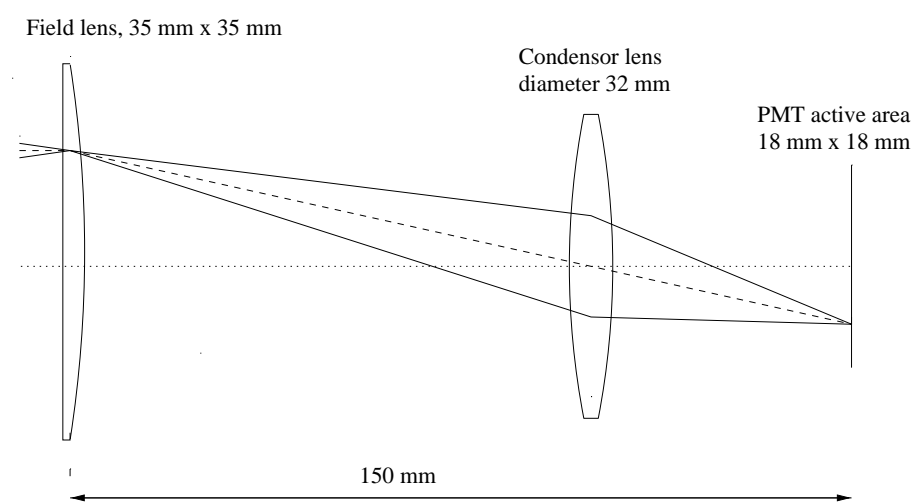

Fig. 6. The optical system for light collection and demagnification. The two rays shown in full line correspond to photons with incident angles of $\pm 100 \mathrm{mrad}$ with respect to the normal incidence (dashed line).

\subsection{COMPASS, Hades and ALICE RICH counters}

The RICH counters of COMPASS (7), Hades (8) and ALICE(9) experiments employ multiwire chambers with a solid CsI layer, evaporated onto the cathode pads, as the photon detector. The material requires a high purity chamber gas, usually methane with water and oxygen content of order ppm, as well as careful 


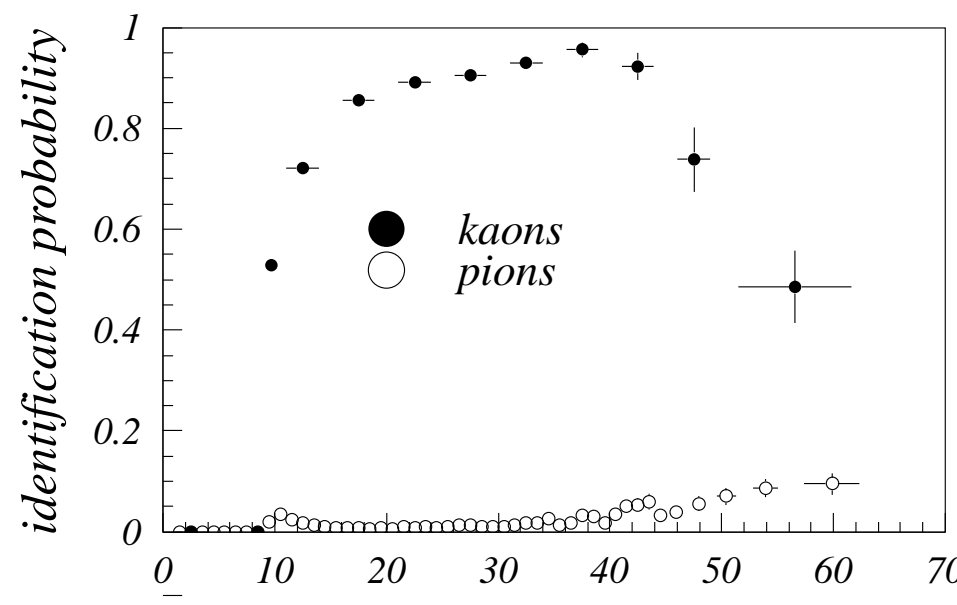

Fig. 7. Particle identification in the HERA-B RICH: efficiencies to identify a kaon and misidentify a pion, as deduced from the $\phi \rightarrow K^{-} K^{+}$and $K_{S} \rightarrow \pi^{-} \pi^{+}$decays.

handling of the photo-cathodes. A lot of R\&D (influence of substrate, thermal conditioning, thickness, evaporation procedure, transfer to the chamber) was needed to make the technique mature $(10 ; 11)$. A special evaporation plant with a sophisticated conditioning facilities and quality assessment controls was constructed at CERN for this purpose. While Compass and Hades RICH counters have been running stably for several years, the ALICE RICH is under construction (12).

In the Compass experiment the central region of the RICH counter was recently upgraded to enable running at higher beam intensities, higher trigger rates and with a nanosecond time resolution for background suppression (13). The wire chamber based photon detector was replaced by an array of multianode PMTs very similar to the HERA-B RICH (Sec. 2.3). The PMTs, again Hamamatsu 16 channel tubes, have quartz windows, and the 7:1 demagnification two-lens light collection system is made of quartz lenses. This results in a larger number of detected photons $(\approx 60$ per ring for $\beta=1$ particles $)$. The measured Čerenkov angle resolution per track is $\approx 0.3 \mathrm{mrad}$, allowing a $2 \sigma$ $\pi / K$ separation at $60 \mathrm{GeV} / c$. The kaon identification efficiency exceeds $90 \%$ with $\pi-K$ misidentification probability at $\approx 1 \%$.

\section{$2.5 \mathrm{LHCb} \mathrm{RICH}$}

In the next generation of $B$ physics experiments at hadron colliders the identification of hadrons will again be essential for both the tagging of $b$-flavour in $C P$ violation and for mixing measurements, as well as for the identification of particles in hadronic final states such as $B \rightarrow \pi^{+} \pi^{-}$. In particular this is so because more channels contribute to the background than in the case of experiments at $\Upsilon(4 s)$ (e.g. $B_{s} \rightarrow K^{+} K^{-}$). In the $\mathrm{LHCb}$ experiment the RICH 
counter is designed to cover $\pi / K$ separation between $1 \mathrm{GeV} / \mathrm{c}$ and $150 \mathrm{GeV} / \mathrm{c}$ (14). The kinematic region covered by a RICH counter, from $p_{\min }$ to $p_{\max }$, depends on the threshold momentum for the lighter of the two particles one wants to separate, and on the resolution in Čerenkov angle (ultimately given by the dispersion in the radiator medium). It turns out that for most radiators $p_{\max } / p_{\min } \approx 4-7(15)$. For the large kinematic interval in the $\mathrm{LHCb}$ experiment one would therefore require three radiators. They are arranged in two counters (14) as shown in Fig. 8.
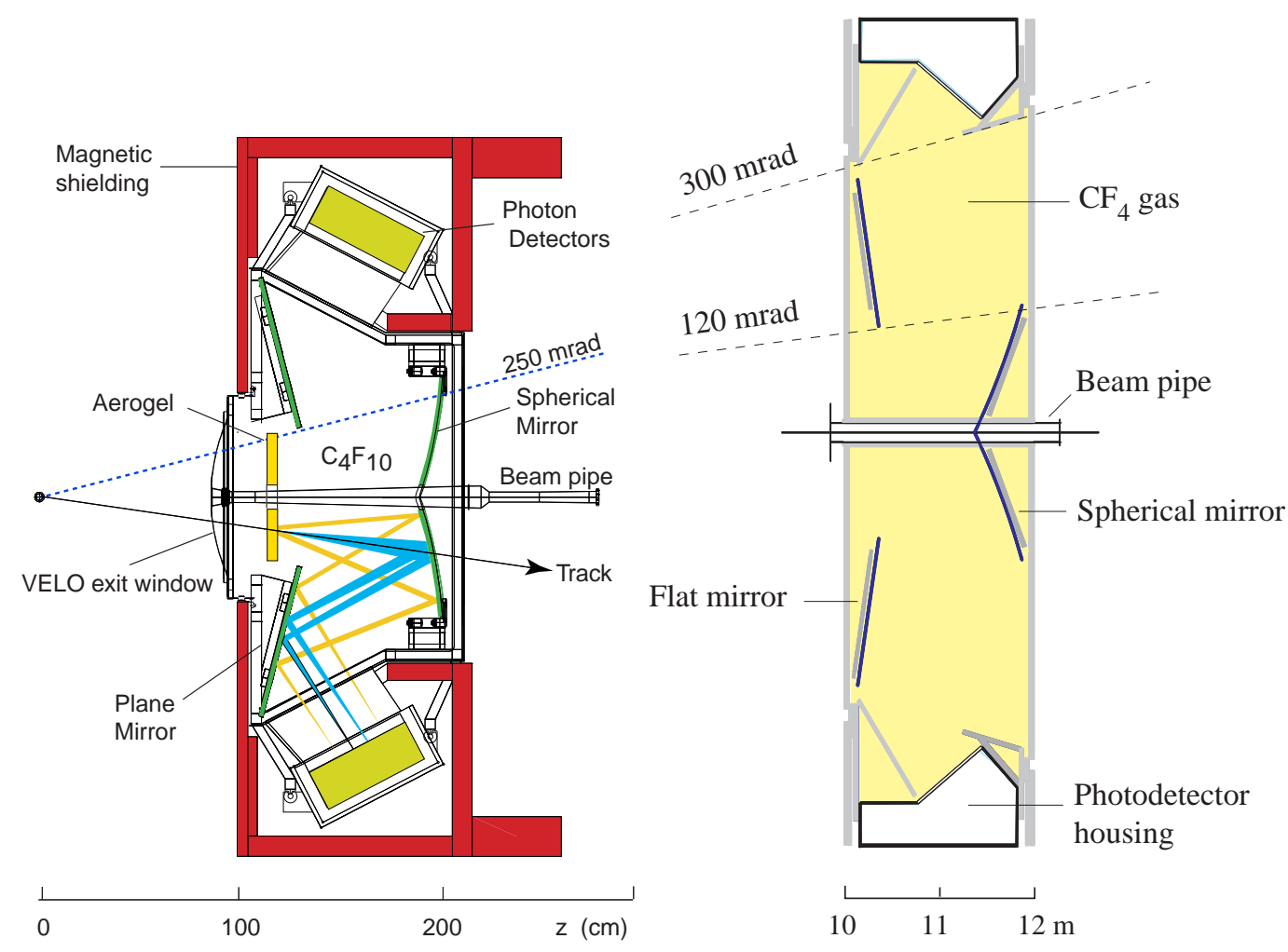

Fig. 8. RICH counters for the LHCb experiment.

Several photon detectors were considered as candidates for the system of RICH counters; a photon detector based on multianode PMTs with a single quartz lens light collection system (16), as well as two types of hybrid photon detectors (HPD). In the latter, a vacuum photosensitive device is combined with charged particle detection in a silicon detector with pixel readout (17). As shown in Fig. 9, photoelectrons are accelerated by the electric field across a potential difference of about $20 \mathrm{kV}$ towards a silicon detector with integrated electronics. The results of beam tests of the final module, developed in collaboration with the DEP company, are very encouraging (18). 


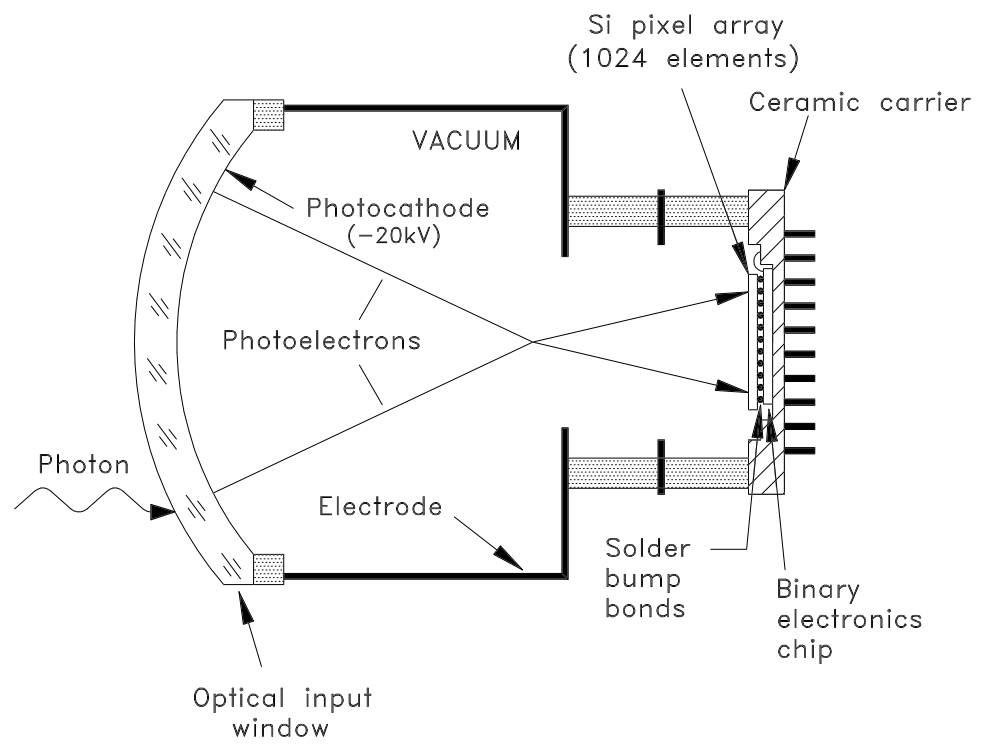

Fig. 9. Hybrid photon detector (HPD) of the LHCb experiment. The outer diameter of the detector is $17 \mathrm{~cm}$.

\section{Recent developments in RICH counters}

For the next round of $B$ physics experiments at upgraded $e^{+} e^{-}$machines ('Super $B$ factories') with luminosities exceeding $10^{35} \mathrm{~cm}^{-2} \mathrm{~s}^{-1}$, considerable improvements of particle identification devices are envisaged in order to cope with higher rates and with more stringent requirements on separation capabilities for rare decay channels.

\subsection{Upgrades at Belle}

Two systems are being considered for the upgrade of the Belle spectrometer (19). For the barrel region a time-of-propagation (TOP) counter is being studied. This is a kind of DIRC counter in which the Čerenkov angle is deduced by measuring one of the coordinates and the time of arrival of Cerenkov photons with high precision (20). Multianode micro-channel plate (MCP) PMTs, including a detector with a GaAsP photocathode, are being investigated (21).

For the end-cap region, a proximity focusing RICH with aerogel as radiator is being tested (Fig. 10). The counter should have a low threshold for pions, and should enable good separation of pions and kaons up to $4 \mathrm{GeV} / c$. Another benefit of such a counter would also be a reasonable $e / \mu / \pi$ separation at low momenta, which is of importance for the studies of rare $B \rightarrow K \ell \ell$ decays.

The feasibility of such a counter was studied in a series of beam tests $(22 ; 23)$. Hamamatsu H8500 ('flat panel') PMTs were used as photon detectors (24). 
As can be seen from Fig. 10, the Čerenkov peak is well pronounced above a small background, mainly coming from Čerenkov photons which were Rayleigh scattered in the radiator. The resolution in the Cerenkov angle measurement $\left(\sigma_{\theta}=14 \mathrm{mrad}\right)$ and the number of detected photons $(N \approx 6$, Fig. 10$)$ agree well with expectations. The resulting resolution per track is about $5.7 \mathrm{mrad}$. Since the difference in Cerenkov angle of pions and kaons is $22 \mathrm{mrad}$ at $4 \mathrm{GeV} / c$, such a counter would allow a good (about $4 \sigma$ ) separation up to the kinematic limit of the experiment.
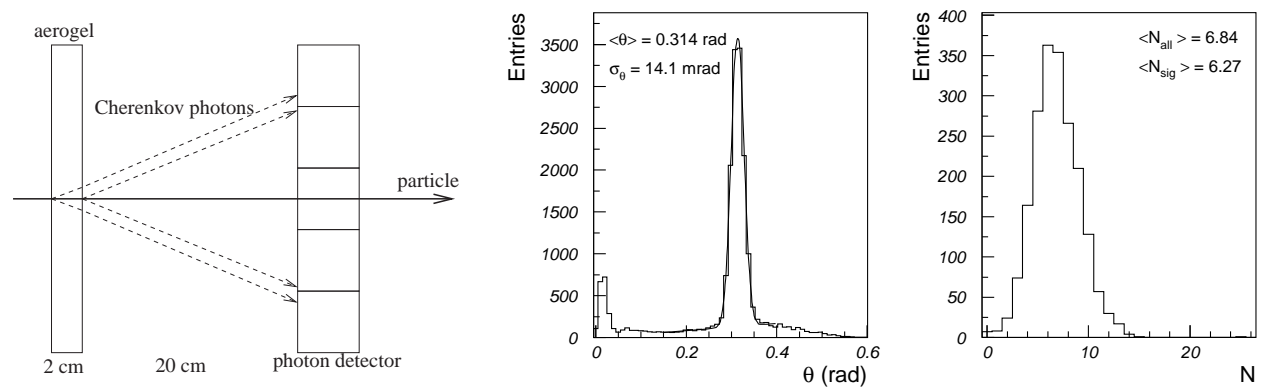

Fig. 10. Proximity focusing RICH for the Belle upgrade in the end-cap region: schematic (a), measured single photon resolution (b) and the number of detected photons (c) for a $2 \mathrm{~cm}$ thick aerogel tile.

The key issue in the performance of a proximity focusing RICH counter is to improve the Čerenkov angle resolution per track $\sigma_{\text {track }}=\sigma_{\theta} / \sqrt{N}$. As it turns out, the optimal thickness is expected to be around $20 \mathrm{~mm}(22 ; 25)$. However, this limitation can be overcome in a proximity focusing $\mathrm{RICH}$ with a nonhomogeneous radiator (25; 26; 27; 28). By judiciously choosing the refractive indices of consecutive aerogel radiator layers, one may achieve overlapping of the corresponding Čerenkov rings on the photon detector (Fig. 11) (28). This represents a sort of focusing of the photons within the radiator, and eliminates or at least considerably reduces the spread due to emission point uncertainty. Note that such a tuning of refractive indices for individual layers is only possible with aerogel, which may be produced with any desired refractive index in the range 1.01-1.07 (29). The dual radiator combination can readily be extended to more than two aerogel radiators. In this case, the indices of aerogel layers should gradually increase from the upstream to the downstream layer.

In Fig. 12, we compare the data for two $4 \mathrm{~cm}$ thick radiators; one with aerogel tiles of equal refractive index $(\mathrm{n}=1.046)$, the other with the focusing arrangement $\left(n_{1}=1.046, n_{2}=1.056\right)$. The improvement is clearly visible. The single photon resolution $\sigma_{\theta}=14.3 \mathrm{mrad}$ for the dual radiator is considerably smaller than the corresponding value for the single refractive index radiator $\left(\sigma_{\theta}=20.7 \mathrm{mrad}\right)$, while the number of detected photons is the same in both cases. 


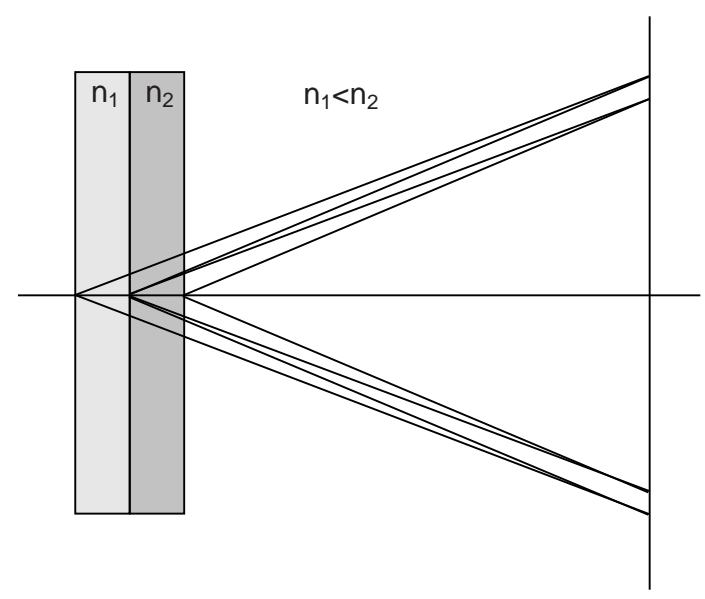

Fig. 11. Proximity focusing RICH with a nonhomogeneous aerogel radiator in the focusing configuration.
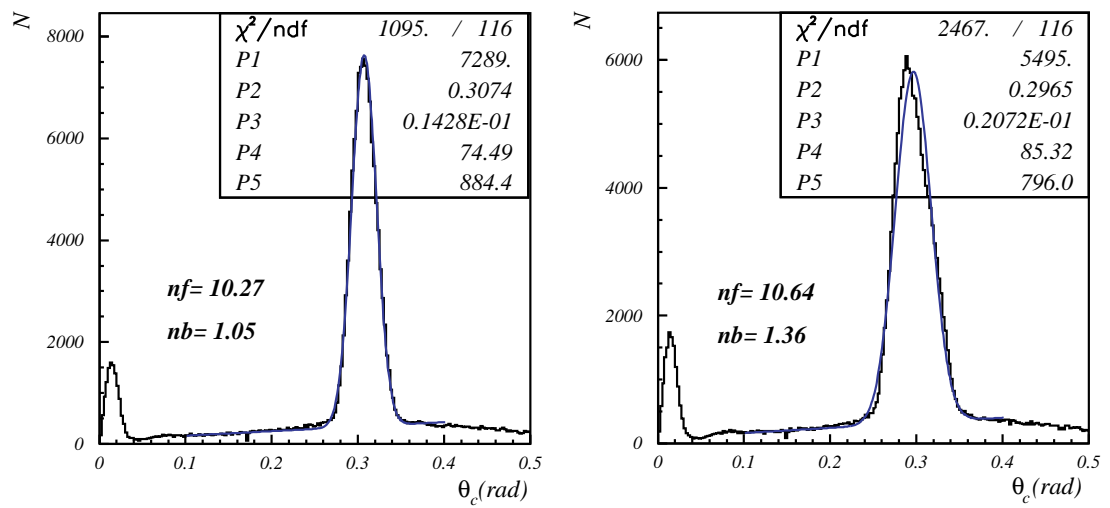

Fig. 12. The accumulated distribution of Cerenkov photon hits depending on the corresponding Čerenkov angle for a $4 \mathrm{~cm}$ homogeneous radiator (right) and a focusing configuration with $n_{1}=1.046, n_{2}=1.056$ (left).

The present R\&D efforts are oriented mainly toward the development of a photon detector which works in the high magnetic field $(1.5 \mathrm{~T})$ of the Belle spectrometer. The Burle 85011 MCP PMT (30), a multichannel device with $8 \times 8$ channels and $6 \mathrm{~mm} \times 6 \mathrm{~mm}$ large pads, has been shown to perform very well as a detector of Cerenkov photons in a RICH counter (31).

Two further detectors are being studied. In a joint effort with the Hamamatsu company a new type of hybrid photon detector of the proximity focusing type is being developed. As the second option, silicon photomultipliers, novel semiconductor photon detectors are being tested $(32 ; 33 ; 34)$. They have several advantages: insensitivity to the high magnetic fields, lower operation voltage, and less material in comparison to the conventional photomultiplier tubes. They also have high peak photon detection efficiency (approx 20\%), high gain of $\approx 10^{6}$ and good time response. Due to their dimensions, they allow compact, light and robust mechanical designs. All this would make them a very promising candidate for a detector of Čerenkov photons in a RICH counter. However, 
due to the serious disadvantage of a very high dark rate $\left(\approx 10^{6} \mathrm{~Hz} / \mathrm{mm}^{2}\right)$, they have up to now never been used in ring imaging Cerenkov detectors, where single photon detection is required at low noise. Because the Cerenkov light is prompt, this problem could in principle be reduced by using a narrow time window $(<10 \mathrm{~ns})$ for signal collection. In addition, it is possible to further increase the signal-to-noise ratio by using light collection systems (35).

\subsection{Focusing DIRC}

As an upgrade of the DIRC counter, a layout with a considerably smaller stand-off box is being considered (36) as shown in Fig. 1. Such a change would significantly reduce the beam related background. However, in order not to degrade the angular resolution, single channel PMTs would have to be replaced by multichannel devices among which the Hamamatsu flat panel 64 channel PMTs and the Burle 64 channel micro-channel plate (MCP) PMTs have been studied. Two further changes are considered which should considerably improve the angular resolution. The uncertainty in the emission point along the track will be eliminated by focusing optics of the expansion volume made of quartz. The chromatic error will be reduced by measuring the time of arrival of Čerenkov photons with a resolution of 50-100 ps, from which the wavelength of each photon can be estimated. The expected resulting total angular error is 4-5mrad per single photon, which makes it possible to achieve an angular resolution of $1.5 \mathrm{mrad}$ per track in principle. While the present BaBar DIRC achieves a $2.7 \sigma \pi / K$ separation at $4 \mathrm{GeV} / c$, the equivalent performance of the upgraded DIRC would be $4.3 \sigma$ at $4 \mathrm{GeV} / c$ for photons traveling a full bar length of 3-4 m.

To demonstrate the capability of eliminating the chromatic error contribution, tests of candidate photon detectors were carried out to evaluate their timing resolution and homogeneity of response (37). By using a pulsed laser diode and dedicated read-out electronics, the time resolution of the MCP PMTs was measured. An excellent time resolution of 30 ps was achieved $(38 ; 39)$. The performance of a focusing DIRC prototype was successfully tested in a test beam (39).

\section{Recent progress in time-of-flight counters}

A proximity focusing RICH counter such as the one discussed in section 3.1 is also a very fast counter, in particular if a micro-channel plate (MCP) PMT is used as the photon detector. With its excellent timing properties, the same device could also serve as a time-of-flight counter and thus supplement other 


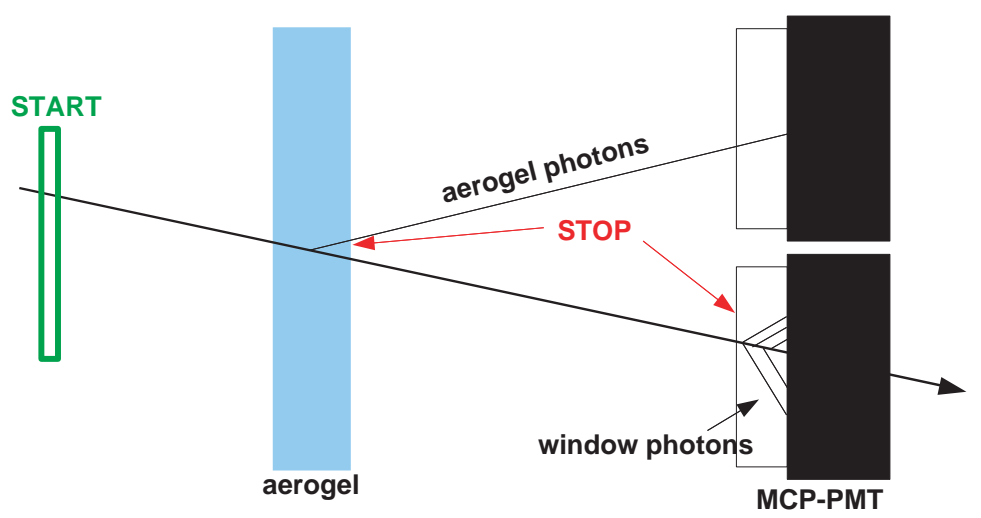

Fig. 13. Schematic view of a combined RICH and TOF counter.
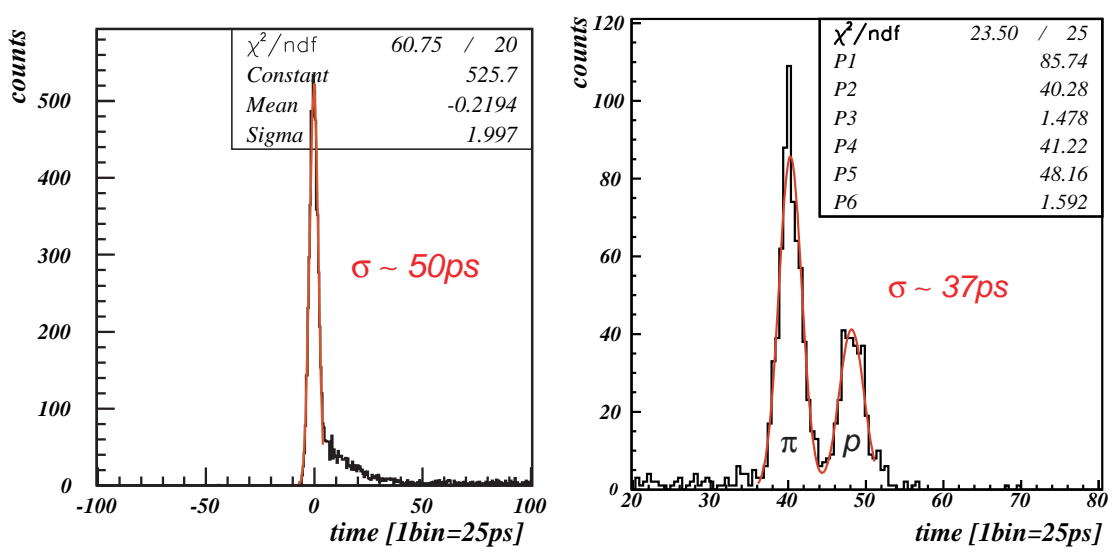

Fig. 14. The distribution of the time of flight as measured by single Cerenkov photons from the aerogel radiator (left), and by Čerenkov photons from the PMT window, shown for $2 \mathrm{GeV} / \mathrm{c}$ pions and protons (right).

identification methods, in particular for low momentum tracks. Čerenkov photons emitted in the radiator medium as well as in the entrance window of the PMT can be used for the time-of-flight measurement (Fig. 13). This allows a positive identification also of particles that would be below the Čerenkov threshold in the aerogel radiator (kaons and protons in the region around $1 \mathrm{GeV}$ ). Consequently, a good separation of kaons and protons would be possible in this region as well.

To test this idea, in a beam test both the hit position and its time were registered with a Burle 85011 MCP PMT (40). The distributions of hits, depending on their time of flight for Čerenkov photons from the aerogel, is plotted in Fig. 14. Fitting this distribution with a Gaussian function yields a standard deviation of about $50 \mathrm{ps}$. For 10 detected hits per track this would correspond to a time-of-flight resolution of 20 ps.

An excellent resolution is also found in the time-of-flight distribution as determined by Cerenkov photons from the PMT window. As can be seen in Fig. 14, the standard deviation of the distribution for pions is $37 \mathrm{ps}$. We can also see 


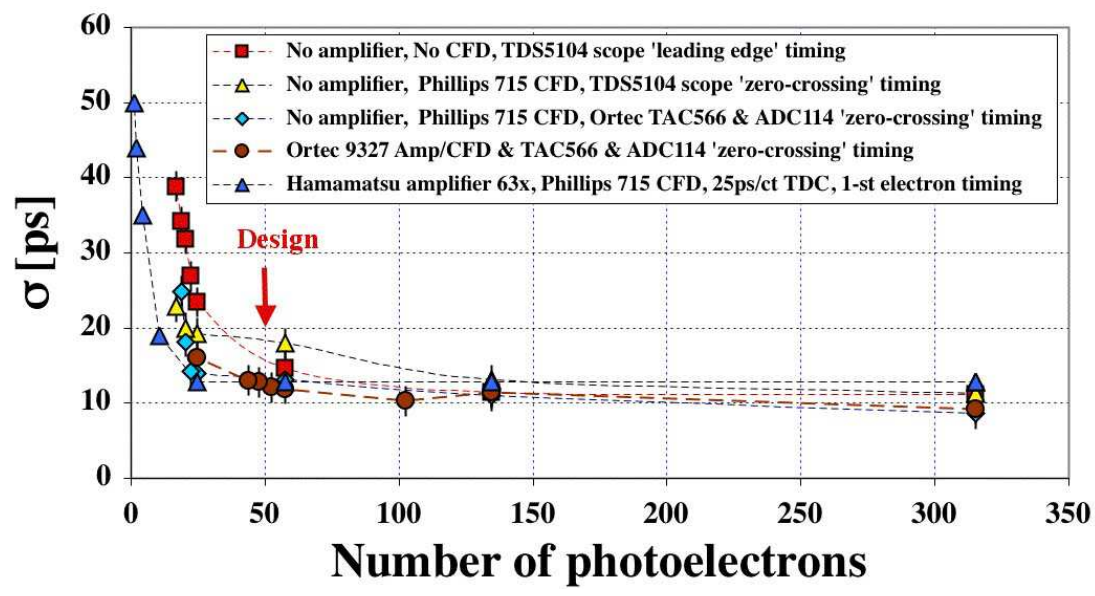

Fig. 15. Timing resolution as a function of number of photoelectrons using various methods for a MCP-PMT with $10 \mu \mathrm{m}$ hole diameter (39).

that pions are clearly separated from protons even in this very compact setup with a very short flight path of $65 \mathrm{~cm}$. In the Belle spectrometer, where the typical flight path is about $2 \mathrm{~m}$, the measured performance would correspond to a $6 \sigma$ separation between pions and kaons at $2 \mathrm{GeV} / \mathrm{c}$, and a $3.5 \sigma$ separation between protons and kaons at $4 \mathrm{GeV} / \mathrm{c}$.

To further improve the time resolution, one could increase the number of photons to about 50 by adding an additional $1 \mathrm{~cm}$ thick quartz radiator in front of the PMT. This idea was tested in a set-up with a somewhat faster version (Burle 85001-501 P01) of the same MCP PMT with $10 \mu \mathrm{m}$ pores (instead of $25 \mu \mathrm{m}$ ), and with a pulsed laser as a source of photons (39). In Fig. 15 the improvement in resolution is clearly observed. By using an even thicker radiator (up to $5 \mathrm{~cm}$ ), a time resolution of 6 ps was observed (41). With such a resolution the time-of-flight counter becomes quite competitive with a RICH counter in the kinematical region up to $4 \mathrm{GeV} / c$.

\section{Summary}

Particle identification devices, in particular the Cerenkov counters of the BaBar and Belle spectrometers have contributed significantly to the rich $B$ physics harvest of the last seven years. Identification of particles in the final state with Čerenkov counters has also contributed to the present understanding of nucleon structure and of the production of heavy quarks in nucleonnucleon collisions. Upgrades are planned to further improve their performance and to allow them to work at even higher event and background rates. New techniques, including a novel focusing radiator concept and new fast photon detectors, have been successfully studied. By using the time of arrival of Čerenkov photons, the dispersion error could be reduced. Furthermore, a 
RICH counter equipped with fast photon detectors can be combined with a time-of-flight or a time-of-propagation counter. Finally, a new type of TOF counter was studied with the same very fast photon detectors. It is expected that these particle identification methods will again play a decisive role in the next generation of precision experiments in $B$ physics.

\section{References}

[1] I. Adam et al., Nucl. Instr. and Meth. A 538 (2005) 281.

[2] T. Sumiyoshi et al., Nucl. Instr. and Meth. A 433 (1999) 385.

[3] T. Iijima et al., Nucl. Instr. and Meth. A 453 (2000) 321.

[4] I. Ariño et al, Nucl. Instr. and Meth. A 516 (2004) 445.

[5] P. Križan et al., Nucl. Instr. and Meth. A 394 (1997) 27; S. Korpar et al., Nucl. Instr. and Meth. A 442 (2000) 316.

[6] D.R. Broemmelsiek, Nucl. Instr. and Meth. A 433 (1999) 136.

[7] E. Albrecht et al., Nucl. Instr. and Meth. A 553 (2005) 215.

[8] K. Zeitelhack et al. [HADES Collaboration], Nucl. Instr. and Meth. A 433 (1999) 201; L. Fabbietti et al. [HADES Collaboration], Nucl. Instr. and Meth. A 502 (2003) 256.

[9] A. Di Mauro et al., IEEE Trans. Nucl. Sci. 52 (2005) 972.

[10] A. Breskin, Nucl. Instr. and Meth. A 371 (1996) 116; F. Piuz, Nucl. Instr. and Meth. A 371 (1996) 95.

[11] H. Hoedlmoser et al., Nucl. Instr. and Meth. A 566 (2006) 338.

[12] Abraham Gallas, paper presented at this Conference.

[13] F. Tessarotto, paper presented at this Conference.

[14] S. Amato et al. (LHCb Collaboration), CERN LHCC 2000-037, CERN, Geneva (2000).

[15] P. Glaessel, Nucl. Instr. and Meth. A 433 (1999) 17.

[16] E. Albrecht et al., Nucl. Instr. and Meth. A 488 (2002) 110.

[17] S. Eisenhardt [LHCb RICH Collaboration], Nucl. Instr. and Meth. A 565 (2006) 234.

[18] G. Vidal-Sitjes et al., paper presented at this Conference.

[19] K. Abe et al. (edited by S. Hashimoto, M. Hazumi, J. Haba, J. W. Flanagan and Y. Ohnishi), "Letter of Intent for KEK Super B Factory", KEK report 2004-04, http://belle.kek.jp/superb/

[20] M. Akatsu et al., Nucl. Instr. and Meth. A 440 (2000) 124.

[21] M. Akatsu et al., Nucl. Instr. and Meth. A 528 (2004) 763.

[22] T. Matsumoto et al., Nucl. Instr. and Meth. A 521 (2004) 367.

[23] P. Križan et al., Nucl. Instr. and Meth. A 533 (2005) 58.

[24] H8500 data sheet, Hamamatsu Photonics K.K.

[25] T. Iijima, S. Korpar et al., Nucl. Instr. and Meth. A 548 (2005) 383.

[26] P. Križan, Super B Factory Workshop, 19-22 Jan 2004, Honolulu, Hawaii, http://www.phys.hawaii.edu/superb04. 
[27] A. Danilyuk et al., Nucl. Instr. and Meth. A 533 (2005) 70.

[28] P. Križan, S. Korpar, T. Iijima, Nucl. Instr. and Meth. A 565 (2006) 457.

[29] I. Adachi et al., Nucl. Instr. and Meth. A 533 (2005) 146.

[30] BURLE 85011 data sheet:

http//www.burle.com/cgi-bin/byteserver.pl/pdf/85011-501.pdf

[31] P. Križan et al., Nucl. Instr. and Meth. A 567 (2006) 124.

[32] P. Buzhan et al., An advanced study of Silicon Photomultiplier, ICFA Instrumentation Bulletin

[33] B. Dolgoshein et al., Nucl. Instr. and Meth. A 563 (2006) 368.

[34] D. Renker, Nucl. Instr. and Meth. A 567 (2006) 48.

[35] R. Pestotnik et al., paper presented at this Conference.

[36] B. Ratcliff, Nucl. Instr. and Meth. A 502 (2003) 211.

[37] C. Field et al., Nucl. Instr. and Meth. A 553 (2005) 96.

[38] J. Va'vra et al., Nucl. Instr. and Meth. A 572 (2007) 459.

[39] J. Va'vra et al., paper presented at this Conference.

[40] S. Korpar et al., Nucl. Instr. and Meth. A 572 (2007) 423.

[41] K. Inami et al., Nucl. Instr. and Meth. A 560 (2006) 303. 